\title{
Ocorrência e controle de lesmas do gênero Omalonyx (Gastropoda, Succineidae), pragas de capim-elefante Pennisetum purpureum (Poaceae) em Rio Preto da Eva, Amazonas
}

\author{
Marcos Vinicius Bastos GARCIA ${ }^{1}$, Janine Oliveira ARRUDA², Daniel Mansur PIMPÃO ${ }^{3}$, Terezinha Batista \\ GARCIA $^{1}$
}

\section{RESUMO}

Lesmas do gênero Omalonyx d'Orbigny, 1837 são hermafroditas, herbívoras, de distribuição neotropical e vivem em plantas aquáticas, nas demais vegetaçóes adjacentes e em solo úmido próximo a ambientes de água doce. No presente trabalho reporta-se a ocorrência atípica de $O$. pattersonae Tillier, 1981 e de Omalonyx sp. em área de terra firme, distante de ambiente aquático. Estas espécies aqui reportadas são simpátricas e devido à alta densidade populacional e prejuízos causados às folhas do capim-elefante Pennisetum purpureum Schumach, são caracterizadas como pragas agrícolas. No período da noite as lesmas se alimentavam das folhas do capim elefante e durante o dia permaneciam escondidas na base do caule, próximo a superfície úmida do solo. A aplicação de cal hidratada sobre agregados de indivíduos de Omalonyx spp foi um método efetivo para o controle das populaçôes. As alteraçóes ambientais dos ecossistemas amazônicos para uso agrícola e/ou urbanizaçáo tem promovido o aumento populacional de espécies que se adaptam a novos habitats e geralmente se tornam pragas de difícil controle.

PALAVRAS-CHAVE: Amazônia, moluscos, controle, cal hidratada

\section{Occurrence and control of Omalonyx slugs (Gastropoda, Succineidae), pests of the elephant-grass Pennisetum purpureum (Poaceae) in Rio Preto da Eva, Amazonas}

\begin{abstract}
Slugs of the genus Omalonyx d'Orbigny, 1837 are hermaphrodites, herbivorous, distributed on Neotropical regions, living on aquatic plants, moist soil and terrestrial vegetation close to freshwater systems. The present paper reports the atypical occurrence of $O$. pattersonae Tillier, 1981 and Omalonyx sp. in an upland area far from any aquatic environment. Both species reported here are sympatric and due to the high populations density and damages caused on leaves of elephant-grass Pennisetum purpureum Schumach, they are recognized as agricultural pests. During the night, the slugs feed on the grass leaves and at the daylight they stayed hidden in the base of stems, near the moist soil surface. The use of hydrated lime distributed over the aggregations of Omalonyx spp. showed to be an effective method to control the populations. The environmental changes in the Amazonian ecosystems for agricultural or urban purposes have promoted the increase of populations of native species that adapt in the new habitat and usually become pests that are difficult to control.
\end{abstract}

KEYWORDS: Amazonia, mollusks, control, hydrated lime

1 Embrapa Amazônia Ocidental, Km 29, Rod. AM 010, Caixa Postal 319, CEP 69011-970, Manaus, AM. E-mail: marcos.garcia@cpaa.embrapa.br

${ }^{2}$ Laboratório de Malacologia, Museu de Ciências e Tecnologia, PUC, Porto Alegre - RS - Brasil. E-mail: arrudajo@gmail.com

${ }^{3}$ Departamento de Biologia Aquática e Limnologia, Pós-graduação, INPA. E-mail: danielpimpao@yahoo.com.br 


\section{INTRODUÇÃO}

As lesmas do gênero Omalonyx d'Orbigny, 1837 são hermafroditas, herbívoras, com uma concha externa reduzida, achatada e unguiforme na região mediana do dorso (Arruda et al. 2006; Arruda 2007). Tem distribuição restrita à região Neotropical, com ocorrência também nas ilhas das pequenas Antilhas, no mar do Caribe (Thiele 1992; Barker 2001; Arruda et al. 2006; Arruda 2007). São animais que ocorrem em ambientes de ecótone sobre plantas aquáticas, nas demais vegetaçốes adjacentes e em solo úmido em planície de inundação de rios, em lagos e brejos. Neste trabalho registra-se a ocorrência atípica de indivíduos de Omalonyx spp. em alta densidade populacional, caracterizando-os como praga agrícola, em plantação de capim-elefante Pennisetum purpureum Schumach, em habitat de terra firme no município de Rio Preto da Eva (Amazonas, Brasil) e sugere-se uma alternativa para o seu controle.

\section{MATERIAL E MÉTODOS}

Foram feitas observações em densa população de lesmas do gênero Omalonyx em uma plantação de capim-elefante (Pennisetum purpureum Schumach) var. Cameron na propriedade agrícola Fazenda J.H., localizada na Rodovia AM-10 a $62 \mathrm{~km}$ de Manaus, no município de Rio Preto da Eva (Amazonas, Brasil). Essas observaçóes foram feitas mensalmente ao longo do primeiro semestre de 2006. A presença das lesmas e sua grande densidade populacional foram denunciadas pelo proprietário da fazenda, que passou a ter prejuízos com a destruição da sua plantação de capimelefante, utilizada na alimentação do gado. Para determinação $\mathrm{da}(\mathrm{s})$ espécie(s) da lesma causadora dos danos, foram coletados espécimes que se encontram depositados na Coleção de Malacologia do Instituto Nacional de Pesquisas da Amazônia (INPA) com o número de tombo 1090. A determinaçáo taxonômica foi realizada a partir do estudo da anatomia do sistema reprodutório de sete dos espécimes coletados.

A intensidade da infestaçáo foi avaliada por meio da contagem direta dos indivíduos. Foram escolhidas aleatoriamente dez touceiras (grupo de oito a 12 plantas) de capim-elefante prétratamento e dez pós-tratamento, que foram examinadas e tiveram todos os espécimes de lesmas contabilizados. Para o controle da praga, foi testado um tratamento utilizando aplicação de cal hidratada. Logo após o corte do capim foram aplicados, a lanço, cerca de $80 \mathrm{~g}$ de cal por touceira. O tratamento foi feito em três etapas, com intervalos de 60 dias (ciclo de corte do capim), durante o período de 180 dias.

\section{RESULTADOS E DISCUSSÃO}

Foram identificadas duas espécies de Omalonyx que estavam causando prejuízo à plantação de capim-elefante, sendo elas O. pattersonae Tillier, 1981 e Omalonyx sp. uma espécie nova (Janine Arruda, informação pessoal, publicação em preparação). As espécies de Omalonyx são determinadas por meio do estudo da anatomia de seus sistemas reprodutórios, sendo impossível diferenciá-las apenas por suas características externas. Omalonyx pattersonae foi anteriormente citada para Antigua, localidade-tipo, e para a Venezuela (Tillier 1981), sendo aqui registrada pela primeira vez para o Brasil.

O comportamento dos indivíduos de Omalonyx variou conforme o horário e as condiçóes meteorológicas do dia. Durante a noite e nos dias chuvosos foram observados indivíduos alimentando-se das folhas de capim-elefante (Figura 1A). Já nos dias ensolarados, permaneciam na base das touceiras, próximos ao solo úmido (Figura 1B). Arruda (2007) observou que Omalonyx convexus (Heynemann, 1868) encontrava-se em diferentes posições no substrato dependendo da hora do dia. Segundo a autora, no início da manhã e final da tarde, quando a temperatura era mais amena, exemplares dessa espécie encontravam-se mais expostos no ambiente, por exemplo sob a regiáo adaxial de folhas de macrófitas; enquanto que nas horas mais quentes do dia, encontravam-se perto das raízes ou na região abaxial das folhas. O comportamento dos indivíduos de Omalonyx observados nesse estudo, i.e., mais ativos nas horas mais amenas do dia e abrigados na base das touceiras nas horas mais quentes, está de acordo com o observado por Arruda (2007).

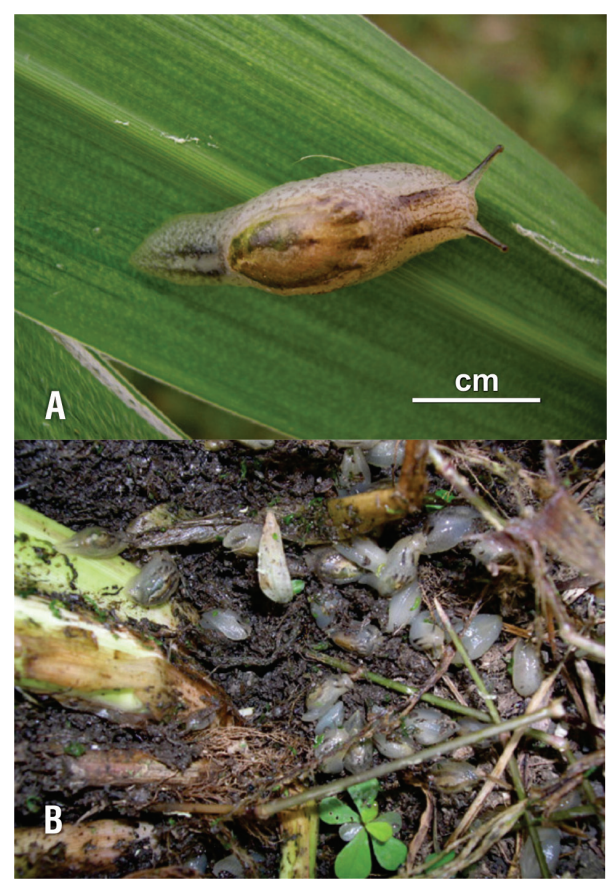

Figura 1 - Espécimes de Omalonyx d'Orbigny, 1837 sobre folha de capimelefante Pennisetum purpureum Schumach (A) e em solo úmido, na base da touceira (B). 
Döring (1873), Parodiz (1963), Hermann e Dundee (1967), Hylton Scott (1971), Tillier (1981), Martinez (1993), Barker (2001) e Arruda et al. (2006) relataram que as lesmas do gênero Omalonyx vivem principalmente sobre macrófitas aquáticas, plantas emergentes ou em solo úmido nas adjacências de ambientes aquáticos. Até o presente momento não há registro na literatura deste grupo vivendo em áreas distantes de ambientes de água doce. Entretanto, neste trabalho relata-se a presença atípica de duas espécies de Omalonyx como pragas de capim-elefante em habitat não inundável e distante de ambiente aquático. Na literatura há registro de espécies do gênero alimentando-se de plantas associadas a seu habitat, ou seja, vegetaçóes adjacentes a ambientes de água doce. Poi de Neiff e Casco (2003) mencionaram que $O$. unguis (d'Orbigny 1837) alimenta-se de Eichhornia crassipes (Mart.) Solms e Salvinia herzogii (de la Sota). Franceschini (2003) descreveu os danos causados por $O$. unguis em folhas de $E$. crassipes.

No levantamento do nível de infestaçáo, antes de se iniciar o tratamento (Figura 2), observou-se de 25 a 94 (média = $62, \mathrm{n}=10$ ) indivíduos de Omalonyx por touceira de capimelefante. Mesmo após o corte do capim-elefante, as lesmas sobreviveram e foram encontradas agregadas próximas às touceiras, abrigando-se nas partes mais úmidas da vegetação adjacente, próxima ao solo.

Vários compostos químicos tem sido sugeridos para o controle químico de gastrópodes terrestres tais como as iscas contendo quelados de alumínio (Henderson et al. 1990); fosfato de ferro (Speiser e Kistler 2002); carbamatos e metaldeído (Henderson e Triebskorn 2002). Embora o composto metaldeído seja considerado o mais eficiente, não

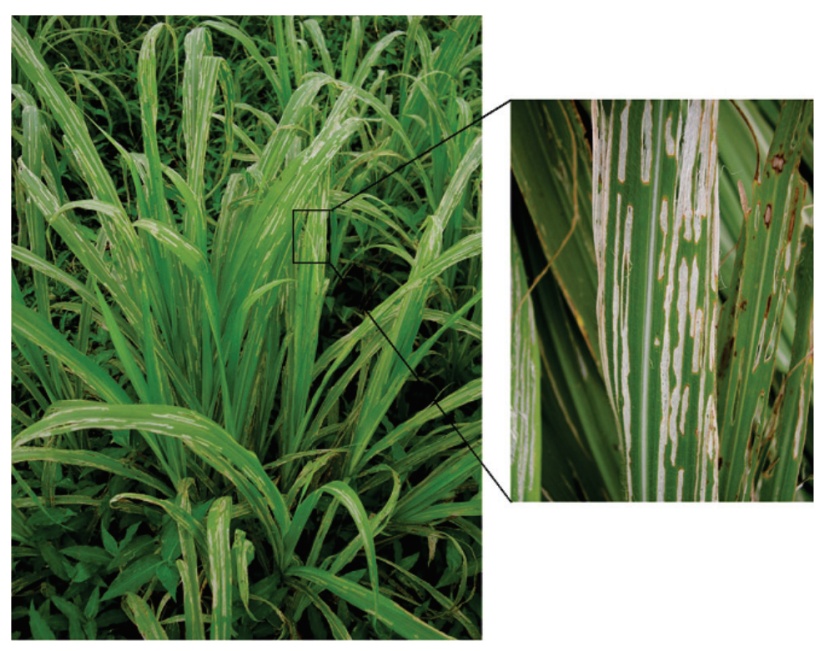

Figura 2 - Danos causados por Omalonyx d'Orbigny, 1837 às folhas do capim-elefante Pennisetum purpureum Schumach. é sempre que há sucesso em sua utilização (Henderson e Triebskorn 2002). Segundo o Sr. Itamar Siqueira, proprietário da fazenda onde foi realizado o presente estudo, o uso de iscas comerciais a base de metaldeído mostrou-se economicamente inviável devido a grande população de lesmas. No Brasil, para o controle de lesmas existem poucas substâncias químicas registradas, tais como o metaldeído, fosfato férrico e metiocarbe. Para o controle de lesmas do gênero Omalonyx não há compostos registrados. Alternativamente, aos agricultores tem sido recomendado o uso de diversos materiais de ação cáustica, como cinzas de madeira e a cal hidratada. Ao contrário da iscas químicas, a utilizaçáo da cal hidratada tem sido sugerida principalmente como barreira para os gastrópodes. No presente estudo, a aplicaçáo de cal hidratada diretamente sobre as agregaçóes de Omalonyx spp., expostas após o corte das touceiras de capim-elefante, mostrouse eficiente no controle destas espécies. Após o período de tratamento o controle foi total em $83 \%$ das touceiras examinadas. Naquelas ainda infestadas, foram observados apenas um ou dois indivíduos de Omalonyx, enquanto que a média de infestação pré-tratamento era de 62 indivíduos. A cal hidratada, além de ter apresentado resultado positivo no controle de Omalonyx, é um produto pouco tóxico para o aplicador e de fácil aquisição no comércio.

Lesmas do gênero Omalonyx foram pela primeira vez mencionadas como pragas agrícolas por Olazarri (1979) em plantação de agriāo (Rorippa nasturtium-aquaticum L.) no Uruguai. Mais recentemente González e Vecco (2004) e Jiménez et al. (2004) reportaram lesmas deste gênero como pragas em plantações de arroz irrigado no Peru. O besouro Cratomorphus sp. (Coleoptera, Lampiridae), considerado como potencial inimigo natural, foi utilizado para controle natural da infestação no Peru (Bardales et al. 2004).

A introdução de espécimes de Omalonyx em pastagens, distante de lagos e planícies de inundaçáo de rios, habitat das espécies desse gênero, ocorreu provavelmente devido ao transporte de exemplares e/ou ovos aderidos às patas dos animais (bois, carneiros, cavalos) que frequentemente são trazidos de áreas inundáveis para pastagens em terra firme. A alteração do habitat dessas lesmas provavelmente acarretou o desequilíbrio populacional que resultou nos danos às folhas de capim-elefante aqui reportadas.

A alteração do ambiente na Amazônia decorrente de atividades agrícolas e da expansão urbana pode favorecer o aumento populacional de espécies anteriormente em equilíbrio com o meio ambiente ou a adaptação destes a novos ambientes, que por sua vez podem vir a tornar-se pragas de difícil controle. Sugere-se que, para o controle destas pragas, sejam avaliadas as alternativas de menor risco para o meio ambiente e para o produtor. 


\section{REFERÊNCIAS}

Arruda, J.O.; Gomes, S.R.; Ramirez, R.; Thomé, J.W. 2006. Morfoanatomia de duas espécies do gênero Omalonyx (Mollusca, Gastropoda, Succineidae) com novo registro para Minas Gerais, Brasil. Biociências, 14 (1): 61-70.

Arruda, J.O. 2007. Sistemática e ecologia de espécies de Omalonyx (Mollusca, Gastropoda, Succineidae) no Estado do Rio Grande do Sul. Dissertação de Mestrado, Pontifícia Universidade Católica do Rio Grande do Sul, Porto Alegre, Rio Grande do Sul. 47 pp.

Bardales, P.R.; Panduro, S.H.; Vecco, G.C.D. 2004. Ciclo biológico y capacidad depredación de Cratomorphus sp. (Coleoptera: Lampiridae) sobre Omalonyx sp. (Pulmonata: succineidae), bajo condiciones de laboratorio en Tarapoto. In: XLVI Convención Nacional de Entomologia. Disponível em: http://www.urkuperu. $\mathrm{org} / \mathrm{sp} /$ res-menes-y-art-culos/index.php. Acesso em: 10 de maio 2010 .

Barker, M.G. 2001. Gastropods on land: phylogeny, diversity and adaptive morphology, p. 1-146. In: Barker, M.G. (Ed.). The biology of terrestrial mollusks. CABI Publishing, New York, New York.

Döring, A. 1873. Bemerkungen über die Mollusken-Fauna der Argentinischen Republik und über einige neue Argentinische Succineen. Malakozoologische Blätter, 11: 49-67, plates II-III.

Franceschini, M.C. 2003. Lesiones producidas por fitófagos en Eichhornia crassipes (Mart.) Solms. In: Comunicaciones Científicas y Tecnológicas. Disponível em: http://www1.unne. edu.ar/cyt/2003/comunicaciones/06-Biologicas/B-034.pdf. Acesso em: 20 de março 2011.

Gonzáles, A.R.; Vecco, G.C.D. 2004. Determinación del consumo y daño foliar que causa la babosa (Pulmonata: Succineidae) en el arroz, variedad capirona, al estado de macollamiento bajo condiciones de laboratorio en Tarapoto - San Martín. In: XLVI Convención Nacional de Entomologia. Disponível em: http:// www.urkuperu.org/sp/res-menes-y-art-culos/index.php. Acesso em: 10 de maio 2010.

Henderson, I.F.; Martin, A.P.; Parker, K.A., 1990. Laboratory and field assessment of a new aluminium chelate slug poison. Crop Protection, 9: 131-134.

Henderson, I.; Triebskorn, R. 2002. Chemical control of terrestrial gastropods, p. 1-31. In: Barker G. M. (ed) Molluscs as crop pests. $\mathrm{CAB}$ International, Wallingford, UK.
Hermann, P. W.; Dundee, D. S. 1967. Notes on Omalonyx. Sterkiana, 28: $1-6$.

Hylton Scott, M. I. 1971. Homalonyx weyrauchi, nueva especie de Tucuman (Gastropoda Succineidae). Neotropica, 17 (52): 12-14.

Jiménez, G.L.E.; Panduro, S.H.; Vecco, G.C.D. 2004. Ciclo biológico y comportamiento alimenticio de la Babosa de los Arrozales (Succineidae: Omalonyx spp.) bajo condiciones de laboratorio en el Bajo Mayo - Tarapoto. In: XLVI Convención Nacional de Entomologia. Disponível em: http://www.urkuperu. $\mathrm{org} / \mathrm{sp} /$ res-menes-y-art-culos/index.php. Acesso em: 10 de maio 2010.

Martínez, R.E. 1993. Nota acerca de la presencia de la babosa Omalonyx (O.) pattersonae Tillier, 1981 (Gastropoda-Pulmonata Succineidae) en Venezuela. Acta Biológica Venezuelica, 14 (2): 65-69.

Olazarri, J. 1979. Los moluscos plaga de los cultivos de "berro" en Salto, Uruguay. Comunicaciones de la Sociedad Malacológica del Uruguay, 5(36): 63-69.

Parodiz, J.J. 1963. Observaciones anatomicas sobre Omalonyx patera Doer., com una nota biografica acerca de Adolfo Doering (18481926). Sterkiana, 12:1-7.

Poi de Neiff, A.S.; Casco, S.L. 2003. Biological agents that accelerate winter decay of Eichhornia crassipes Mart. Solms. in northeastern Argentina, p. 127-144. In: Thomas, S.M., Bini, L.M. (Eds.) Ecologia e Manejo de macrófitas aquáticas. Editora da Universidade Estadual de Maringá, Maringá, Paraná.

Speiser, B.; Kistler, C. 2002. Field tests with a molluscicide containing iron phosphate, Crop Protection, 21: 389-394.

Thiele, J. 1992. Handbook of Systematic Malacology, part II: Gastropoda: Opisthobranchia and Pulmonata. Scientific editors of translation: Rüdiger Bieler and Paula M. Mikkelsen. Smithsonian Institution Libraries and The National Science Foundation, Washington, DC, USA [do original em alemão Handbuch der systematischen Weichtierkunde]. p. 471-782.

Tillier, S. 1981. South American and Juan Fernández succineid slugs (Pulmonata). Journal of Molluscan Studies, 47: 125-146.

Recebido em 06/01/2011

Aceito em 20/05/2011 